\title{
Chromatic Numbers of Products of Graphs: The Directed and Undirected Versions of the Poljak-Rödl Function
}

\section{Claude Tardif and David Wehlau}

DEPARTMENT OF MATHEMATICS AND COMPUTER SCIENCE ROYAL MILITARY COLLEGE OF CANADA

PO BOX 17000, STATION "FORCES"

KINGSTON, ONTARIO K7K 7B4 CANADA

E-mail: Claude.Tardif@rmc.ca

E-mail:wehlau@rmc.ca

Received July 29, 2003; Revised December 21, 2004

Published online 5 August 2005 in Wiley InterScience(www.interscience.wiley.com). DOI 10.1002/jgt.20117

Abstract: Let $f(n)=\min \{\chi(G \times H): G$ and $H$ are $n$-chromatic digraphs $\}$ and $g(n)=\min \{\chi(G \times H): G$ and $H$ are $n$-chromatic graphs $\}$. We prove that $f$ is bounded if and only if $g$ is bounded. (c) 2005 Wiley Periodicals, Inc. J Graph Theory 51: 33-36, 2006

Keywords: categorical product; Hedetniemi's conjecture; chromatic number; Poljak-Rödl function

Contract grant sponsor: Natural Science and Engineering Research Council of Canada (NSERC) Discovery grants, Research Program (ARP).

(c) 2005 Wiley Periodicals, Inc. 


\section{INTRODUCTION}

The categorical product $\vec{G} \times \vec{H}$ of two directed graphs $\vec{G}$ and $\vec{H}$ is the directed graph with vertex set $V(\vec{G}) \times V(\vec{H})$ and $\operatorname{arcs}\left(\left(u_{1}, u_{2}\right),\left(v_{1}, v_{2}\right)\right)$ for all arcs $\left(u_{1}, v_{1}\right)$ of $\vec{G}$ and $\left(u_{2}, v_{2}\right)$ of $\vec{H}$. Undirected graphs can be viewed as directed graphs with symmetric arcs hence the definition of the product extends readily. Hedetniemi's conjecture [4] states that the identity $\chi(G \times H)=\min \{\chi(G)$, $\chi(H)\}$ holds for all undirected graphs $G$ and $H$. It is known to hold when $\min \{\chi(G), \chi(H)\} \leq 4$ (see [2]) or when $G$ and $H$ belong to special classes of graphs (see $[7,10]$ for recent surveys).

Proper colorings and chromatic numbers are usually defined for undirected rather than directed graphs, but since the orientation of the edges does not affect the constraints, we can just define the chromatic number of a directed graph as that of the graph obtained by ignoring the orientation of its edges. However, note that the orientation of the arcs of the factors determines the structure of a product, and its chromatic number. Thus it is possible to extend the investigation of chromatic numbers of products to directed graphs. In this respect, Poljak and Rödl [6] were the first to note that the inequality $\chi(\vec{G} \times \vec{H}) \leq \min \{\chi(\vec{G}), \chi(\vec{H})\}$ can be strict for directed graphs; examples already exist among tournaments, that is, orientations of complete graphs. The same authors introduced the functions

$$
\begin{aligned}
& f(n)=\min \{\chi(\vec{G} \times \vec{H}): \vec{G} \text { and } \vec{H} \text { are n-chromatic directed graphs }\}, \\
& g(n)=\min \{\chi(G \times H): G \text { and } H \text { are n-chromatic undirected graphs }\} .
\end{aligned}
$$

Thus, Hedetniemi's conjecture states that $g(n)=n$ for all $n$. In general, we have $f(n) \leq g(n) \leq n$; it is known that $g(n)=n$ for $n \leq 4$ ([2]), $f(n)=n$ for $n \leq 3$ and $f(n)<n$ for all $n \geq 4([1,6])$.

Perhaps surprisingly, it is not even known whether $f(n)$ and $g(n)$ grow without bound with $n$. Poljak and Rödl showed that if $f$ is bounded above, then the bound is at most 4, and used this result to show that if $g$ is bounded above, then the bound is at most 16. In [5] (see also [10]), these bounds have been improved: if $f$ is bounded above, then the bound is 3 , and if $g$ is bounded above, then the bound is at most 9. Thus, either we have $f(n)=\min \{3, n\}$ for all $n$, or $\lim _{n \rightarrow \infty} f(n)=\infty$. Of course if $f$ is unbounded, then $g$ also unbounded. In this note, we prove the converse:

Theorem 1. If $f$ is bounded, then so is $g$.

It has been shown in [8] that for all $\epsilon>0$, there exists $n_{\epsilon}$ such that for all $n \geq n_{\epsilon}$ there exist $n$-tournaments $S_{n}, T_{n}$ such that $\chi\left(S_{n} \times T_{n}\right) \leq((2 / 3)+\epsilon) n$ (thus $\lim _{n \rightarrow \infty} f(n) / n \leq 2 / 3$ ). In [1,9], we find examples of directed graphs $\vec{G}_{n}$, $\vec{H}_{n}$ such that $\chi\left(\vec{G}_{n}\right)=n, \chi\left(\vec{H}_{n}\right)=4$, and $\chi\left(\vec{G}_{n} \times \vec{H}_{n}\right)=3$. Thus the world of directed graphs offers many examples of product graphs with interesting chromatic properties. The world of undirected graphs offers some confirmations 
of Hedetniemi's conjecture, and through their proofs, intriguing links with the topological aspects of graph coloring (see [2, 7, 10]). Theorem 1 above shows that these two facets of product graph coloring are linked together. The remainder of the paper is devoted to its proof.

\section{PROOF OF THEOREM 1}

Let $K_{n}$ denote the complete graph on $n$ vertices. For a directed graph $\vec{G}$, the exponential digraph $K_{n}^{\vec{G}}$ is the directed graph whose vertices are all functions from the vertex set of $\vec{G}$ to that of $K_{n}$, where two functions $f, g$ are joined by an arc if $f(u) \neq g(v)$ for every arc $(u, v)$ of $\vec{G}$. For any directed graph $\vec{H}$, there is a natural correspondence between the proper $n$-colorings of $\vec{G} \times \vec{H}$ and the arcpreserving maps from $\vec{H}$ to $K_{n}^{\vec{G}}$ : If $c: \vec{G} \times \vec{H} \mapsto K_{n}$ is a proper coloring, then the map $\phi_{c}: \vec{H} \mapsto K_{n}^{\vec{G}}$ defined by $\phi_{c}(v)=f_{v}$, where $f_{v}(u)=c(u, v)$ is arc-preserving. Conversely, if $\phi: \vec{H} \mapsto K_{n}^{\vec{G}}$ is arc-preserving, then $c_{\phi}: \vec{G} \times \vec{H} \mapsto K_{n}$ defined by $c_{\phi}(u, v)=f_{v}(u)$, where $f_{v}=\phi(v)$ is a proper coloring. Thus we have $\chi(\vec{G} \times \vec{H}) \leq n$ if and only if there exists an arc-preserving map from $\vec{H}$ to $K_{n}^{\vec{G}}$. In particular, if $\chi(\vec{H})>\chi\left(K_{n}^{\vec{G}}\right)$, then $\chi(\vec{G} \times \vec{H})>n$. These and other properties of exponential digraphs are discussed in $[2,3,7,10]$.

Suppose that the function $f$ is bounded. Then by the results of $[5,10]$, we have $f(n) \leq 3$ for all $n$. Let $\left\{\overrightarrow{\boldsymbol{G}}_{k}, \vec{H}_{k}\right\}_{k>4}$ be a sequence of pairs of directed graphs such that $\chi\left(\vec{G}_{k}\right)=\chi\left(\vec{H}_{k}\right)=k$ and $\chi\left(\vec{G}_{k} \times \vec{H}_{k}\right)=3$. We construct a sequence $\left\{A_{n}, B_{n}\right\}_{n \geq 10}$ of pairs of graphs such that $\chi\left(A_{n}\right)=\chi\left(B_{n}\right)=n$ and $\chi\left(A_{n} \times B_{n}\right) \leq 9$ as follows.

For a fixed $n \geq 10$, put

$$
m=\max \left\{\chi\left(K_{n-1}^{\vec{G}_{n}}\right), \chi\left(K_{n-1}^{\vec{H}_{n}}\right)\right\}+1 .
$$

Then $\chi\left(\vec{G}_{m}\right)>\chi\left(K_{n-1}^{\vec{G}_{n}}\right)$ whence $\chi\left(\vec{G}_{n} \times \vec{G}_{m}\right)=n$. Let $A_{n}$ be the graph obtained by ignoring the orientation of the arcs of $\vec{G}_{n} \times \vec{G}_{m}$. Similarly, for the directed graph $\vec{H}_{m}^{-}$obtained from $\vec{H}_{m}$ by reversing the orientation of the arcs, we have $\chi\left(\vec{H}_{m}^{-}\right)>\chi\left(K_{n-1}^{\vec{H}_{n}}\right)$ whence $\chi\left(\vec{H}_{n} \times \vec{H}_{m}^{-}\right)=n$. Let $B_{n}$ be the graph obtained by ignoring the orientation of the arcs of $\vec{H}_{n} \times \vec{H}_{m}^{-}$.

Fact. $\chi\left(A_{n} \times B_{n}\right) \leq 9$.

Proof of Fact. Let $c_{n}: \vec{G}_{n} \times \vec{H}_{n} \mapsto\{0,1,2\}$ and $c_{m}: \vec{G}_{m} \times \vec{H}_{m} \mapsto\{0,1,2\}$ be proper 3-colorings of $\vec{G}_{n} \times \vec{H}_{n}$ and $\vec{G}_{m} \times \vec{H}_{m}$, respectively. Consider the function $\hat{c}: A_{n} \times B_{n} \mapsto\{0,1,2\} \times\{0,1,2\}$ defined by $\hat{c}((u, v),(w, x))=$ $\left(c_{n}(u, w), c_{m}(v, x)\right)$; we will show that $\hat{c}$ is a proper coloring of $A_{n} \times B_{n}$.

Let $\left[((u, v),(w, x)),\left(\left(u^{\prime}, v^{\prime}\right),\left(w^{\prime}, x^{\prime}\right)\right)\right]$ be an edge of $A_{n} \times B_{n}$. Then $[(u, v)$, $\left.\left(u^{\prime}, v^{\prime}\right)\right]$ is an edge of $A_{n}$ and $\left[(w, x),\left(w^{\prime}, x^{\prime}\right)\right]$ is an edge of $B_{n}$. By symmetry, we can suppose that $\left((u, v),\left(u^{\prime}, v^{\prime}\right)\right)$ is an arc of $\vec{G}_{n} \times \vec{G}_{m}$ and we need to consider two possibilities: 
(i) If $\left((w, x),\left(w^{\prime}, x^{\prime}\right)\right)$ is an arc of $\vec{H}_{n} \times \vec{H}_{m}^{-}$, then $\left(w, w^{\prime}\right)$ is an arc of $\vec{H}_{n}$, so that $\left((u, w),\left(u^{\prime}, w^{\prime}\right)\right)$ is an arc of $\vec{G}_{n} \times \vec{H}_{n}$, whence $c_{n}(u, w) \neq c_{n}\left(u^{\prime}, w^{\prime}\right)$.

(ii) Otherwise $\left(\left(w^{\prime}, x^{\prime}\right),(w, x)\right)$ is an arc of $\vec{H}_{n} \times \vec{H}_{m}^{-}$, thus $\left(x^{\prime}, x\right)$ is an arc of $\vec{H}_{m}^{-}$, so that $\left(x, x^{\prime}\right)$ is an arc of $\vec{H}_{m}$ and $\left((v, x),\left(v^{\prime}, x^{\prime}\right)\right)$ is an arc of $\vec{G}_{m} \times \vec{H}_{m}$, whence $c_{m}(v, x) \neq c_{m}\left(v^{\prime}, x^{\prime}\right)$.

So in either case, we have $\hat{c}((u, v),(w, x)) \neq \hat{c}\left(\left(u^{\prime}, v^{\prime}\right),\left(w^{\prime}, x^{\prime}\right)\right)$. This shows that $\hat{c}$ is a proper vertex coloring of $A_{n} \times B_{n}$ using the nine colors in $\{0,1,2\} \times\{0,1,2\}$. This concludes the proof of the fact.

Thus, $\left\{A_{n}, B_{n}\right\}_{n>10}$ is a sequence of pairs of graphs such that $\chi\left(A_{n}\right)=$ $\chi\left(B_{n}\right)=n$ and $\chi\left(A_{n} \times B_{n}\right) \leq 9$, which shows that $g(n) \leq 9$ for all $n$. This concludes the proof of Theorem 1 .

\section{REFERENCES}

[1] S. Bessy and S. Thomassé, The Categorical Product of two 5-chromatic digraphs can be 3-chromatic, preprint 2004, 2 pages MS, Discrete Math, to appear.

[2] M. El-Zahar and N. Sauer, The chromatic number of the product of two 4chromatic graphs is 4, Combinatorica 5 (1985), 121-126.

[3] R. Häggkvist, P. Hell, D. J. Miller, and V. Neumann-Lara, On multiplicative graphs and the product conjecture, Combinatorica 8 (1988), 71-81.

[4] S. H. Hedetniemi, Homomorphisms of graphs and automata, University of Michigan Technical Report 03105-44-T, 1966.

[5] S. Poljak, Coloring digraphs by iterated antichains, Comment Math Univ Carolin 32 (1991), 209-212.

[6] S. Poljak and V. Rödl, On the arc-chromatic number of a digraph, J Combin Theory Ser B 31 (1981), 339-350.

[7] N. Sauer, Hedetniemi's conjecture-A survey, Discrete Math 229 (2001), 261-292.

[8] C. Tardif, Chromatic numbers of products of tournaments: Fractional aspects of Hedetniemi's conjecture, Graphs, Morphisms and Statistical Physics, 171-175 DIMACS Ser Discrete Math Theoret Comput Sci, 63, Amer Math Soc, Providence, RI, 2004.

[9] C. Tardif, The fractional chromatic number of the categorical product of graphs, preprint, 9 pages MS, Combinatorica, to appear.

[10] X. Zhu, A survey on Hedetniemi's conjecture, Taiwanese J Math 2 (1998), $1-24$. 